$56^{\text {ème }}$ Congrès de la SFMBCB, 03005 (2011)

DOI: $10.1051 / \mathrm{sfmbcb} / 20115603005$

(C) Owned by the authors, published by EDP Sciences, 2011

\title{
Une nouvelle technique d'expansion transversale de la crête alvéolaire mandibulaire en deux temps : illustration par un cas clinique
}

\author{
Vinay N, Torres JH \\ Service d'Odontologie, CHU, Montpellier, France \\ nv_blondin@yahoo.fr
}

Dans les cas où une insuffisance de volume osseux ne permet pas la mise en place d'implants, l'expansion ou augmentation transversale des crêtes alvéolaires constitue une alternative aux greffes osseuses d'apposition, à la régénération osseuse guidée ou à la distraction transversale. Récemment, cette technique a été améliorée grâce au développement des instruments piézoélectriques (Peivandi et al. 2007). Au maxillaire, l'expansion transversale est aisée. Elle est basée sur la plasticité de l'os obtenu après corticotomie et l'utilisation d'expanseurs de crêtes. Cette technique pose davantage de problèmes à la mandibule. En effet, du fait de l'épaisseur des corticales mandibulaires, l'os offre peu de plasticité et le risque de fracture est élevé, situation qui compromet la stabilité primaire des implants, et augmente le risque de nécrose et de résorption du fragment luxé. Une nouvelle technique en 2 temps est présentée, à travers un cas clinique : il s'agit d'une pré-découpe d'un volet, avec mise en place différée des implants sans décollement d'un lambeau, dans le but de respecter l'apport vasculaire d'origine périostée.

Une femme de 45 ans présente un édendement postérieur terminal mandibulaire droit. Un traitement implantaire est envisagé pour remplacer 45 et 46 . L'examen clinique et le scanner montrent une crête très mince : le volume osseux est insuffisant pour placer des implants selon la technique classique. Au cours d'une première intervention, un volet osseux cortical vestibulaire, situé en regard du site à implanter, est isolé par un trait "haut" d'ostéotomie piézoélectrique qui fend la crête sagittalement, 2 traits verticaux "de décharge", puis un trait horizontal "bas" reliant les incisions verticales et délimitant un rectangle. Ce volet n'est pas luxé ; le lambeau est simplement suturé. Au cours d'une seconde intervention à J+21, une incision crestale isolée (sans incision de décharge et surtout sans décollement de lambeau vestibulaire), est pratiquée au dessus du trait d'ostéotomie crestal réalisé préalablement. On introduit une lame malléable coudée à $90^{\circ}$ dans le trait d'ostéotomie crestal, et un mouvement de rotation permet d'écarter les 2 pièces osseuses, le fragment cortical "pré-découpé" lors de la première intervention est luxé. Après un léger forage, les implants (3I, diamètre $3.5 \mathrm{~mm}$ ) sont insérés et une vis d'ostéosynthèse stabilise fermement le volet vestibulaire à la corticale linguale, participant ainsi à la stabilité primaire des implants. Aucun biomatériau de substitution osseuse n'est mis en place et le lambeau lingual est libéré pour permettre une suture muqueuse sans tension.

Ce protocole d'expansion de crête en 2 temps permet de ménager la vascularisation du volet osseux lors de sa luxation, ce qui pourrait contribuer d'une part à réduire les complications liées à la trophicité et d'autre part à réduire la résorption souvent observée dans les greffes d’apposition à la mandibule. Cette technique demande à être évaluée. 